\title{
The Fundamental Constraint on the evolution of culture
}

\author{
GRANT RAMSEY \\ Department of Philosophy, Duke University, Durham, NC, 27708, USA \\ (E-mail: grant.ramsey@duke.edu)
}

Received 10 November 2005; accepted in revised form 6 June 2006

Key words: Culture, Constraint, Evolution, Imitation, Innovation, Social learning

\begin{abstract}
This paper argues that there is a general constraint on the evolution of culture. This constraint - what I am calling the Fundamental Constraint - must be satisfied in order for a cultural system to be adaptive. The Fundamental Constraint is this: for culture to be adaptive there must be a positive correlation between the fitness of cultural variants and their fitness impact on the organisms adopting those variants. Two ways of satisfying the Fundamental Constraint are introduced, structural solutions and evaluative solutions. Because of the limitations on these solutions, this constraint helps explain why there is not more culture in nature, why the culture that does exist has the form it has, and why complex, cumulative culture is restricted to the human species.
\end{abstract}

\section{Introduction}

It might seem that it is generally better to be cultured, ${ }^{1}$ that is, that individuals would be better off if they possessed the ability and disposition to socially transmit information. After all, individuals of a species exhibiting the social transmission of information can potentially acquire information at a lower cost, more reliably, and faster. Instead of risking dangerous trial and error learning - learning, for example, which species are predators and what to do to avoid them - individuals can readily learn this invaluable information from their conspecifics.

But being cultured also has its costs. There are reasons why learning individually or being "hard wired" might be preferable to social learning. For example, Boyd and Richerson (1985) point out that if environmental change is too fast or too slow, cultural learning can be deleterious: an individual in a rapidly changing environment who gets old information from a conspecific is likely to be worse off than if it used trial and error learning. Similarly, in a rather stable environment it is likely to be advantageous to be hard wired to

\footnotetext{
1 For the purposes of this paper, I will employ a broad definition of 'culture', which includes all non-genetic forms of inheritance. This is not to imply that I take this to be the best definition of culture. Instead, I am applying this definition because of its simplicity and clarity. Other definitions of culture would require substantial support and clarification, which is beyond the scope of this paper.
} 
behave in fixed ways instead of learning (both because there is an inherent cost to learning and because conspecific models can be unreliable or deceptive).

There are also many traits - such as the ability to imitate and innovate whose absence can act to constrain the evolution of culture. ${ }^{2}$ Recognizing that culture is adaptive in some ways, but maladaptive or constrained in other ways, leads one to ask whether there is an overarching framework in which to understand the evolution of culture. In this paper, I introduce a general constraint - what I am calling the Fundamental Constraint on the evolution of culture - which provides a way of explaining why culture has arisen in some lineages but not others, as well as explaining why culture, especially complex, cumulative culture is not more common than it is.

\section{Constraints on the evolution of culture}

Before introducing the Fundamental Constraint, I will briefly map out the diversity of constraints on the evolution of culture. There are six dimensions in which something can act as a constraint: the Transformation, Reception, Implementation, Propagation, Production, and Evaluation (TRIPPE for short) of cultural variants can be constrained. The presence of any one of these constraints can eliminate the adaptiveness (or even possibility) of culture. Let's look at some examples of constraints on culture and see which TRIPPE dimensions they occupy.

\section{Imitation}

Many forms of culture require imitation $^{3}$ and the ability to imitate can be cognitively and perceptually demanding. A lack of culture - certain kinds of culture, at least - might therefore be explained in terms of a lack of certain

\footnotetext{
2 Both 'evolution' and 'culture' are polysemous. Thus, the phrase 'cultural evolution' is multiply ambiguous. The evolution of a trait can mean the origin of a trait or the change of a preexisting trait. 'Culture' can refer to an ensemble of cultural variants (e.g., American culture), particular cultural variant types (e.g., driving on the right side of the road), a particular cultural variant token (e.g., Martha's way of tying her shoelaces, learned from her mother), or to a cultural system as a whole (e.g., songbirds exhibit culture but roaches do not). In producing a framework in which to understand the evolution of culture, I am chiefly concerned with evolution in the sense of origin (though maintenance is also important) and culture in the sense of a cultural system as a whole. Thus, a constraint on the evolution of culture (in this sense) concerns the origin (and maintenance) of cultural systems. The constraint I propose shows what it takes for cultural systems (as a whole) are to be adaptive, i.e., why it would be advantageous to learn how to behave from conspecifics instead of learning individually or being hard wired.

3 There is much debate whether "true" imitation (Thorpe 1963) occurs in nature and some have even argued that the human imitative ability is unique and different in kind from the copying found among other animals, and that this ability is necessary for complex, cumulative culture (Tomasello et al. 1993; Tomasello 1999). For a discussion of the concept of imitation see Whiten and Ham (1992) and Byrne and Russon (1998).
} 
kinds of imitation. Imitation minimally requires that an individual observe another individual and (thereby) produce a behavior that correlates with the observed behavior. Thus, the TRIPPE dimensions affected by this constraint could be either reception or implementation (or both). If an individual cannot perceive the behaviors of others or store this information (reception), imitation is impossible. Likewise, if the individual cannot put this information to use (implementation), imitation is impossible.

\section{Innovation}

Even if members of a species are able to imitate flawlessly, it does not follow that imitating others is adaptive. Although a species might be able to imitate behavioral variants of others, there could be a paucity of good behavioral variants available to be imitated. One reason for this is that the species might not have a propensity to innovate. ${ }^{4}$ If one's conspecifics rarely produce beneficial innovations, then it will be better not to copy them. There are a number of reasons why a species might not produce beneficial innovations. One is that the species lacks neurological structures labile enough to produce behavioral novelty, or that the novel behaviors that are produced tend to be worse than the status quo, e.g., they tend to be mistakes. This is an example of a constraint on transformation or production (or both).

\section{Social structure}

Even if members of a species are able to imitate flawlessly and regularly produce beneficial innovations, culture may still not evolve. This is because the organisms need to be sufficiently social for cultural variants to be able to spread among the members. Social structure is an example of a constraint on propagation. ${ }^{5}$

\section{Discrimination}

Even if individuals are able to innovate and imitate and there is an amenable social structure, if the individuals are not disposed to adopt the good variants, then it may not be good to be cultured. (As we will see below, although it is not always necessary that individuals are able to make such discriminations, there is a large class of cases in which it is necessary.) Individuals can make these discriminations to determine the variants that they will adopt or the variants

\footnotetext{
4 For a discussion of the concept of innovation, see Ramsey et al. (submitted) and Laland and Reader (2003).

5 For an example from anthropology, see Henrich's (2004) model of the structure of social transmission and the explanation of maladaptive losses of cultural variants in the case of Tasmania.
} 
others (e.g., their offspring) will adopt. ${ }^{6}$ This is an example of a constraint on evaluation.

\section{The Fundamental Constraint}

The constraints listed above can each act to keep a species from evolving a cultural system. And they can help to explain why some species have never evolved a cultural system, while others have. But what is lacking from these explanations is an overarching framework, a way to say, in general, what it takes for a cultural system to be adaptive. For this, we need to understand what I am calling the Fundamental Constraint on the evolution of culture.

The Fundamental Constraint is this: for culture to be adaptive there must be a positive correlation between the fitness of cultural variants and their fitness impact on the organisms adopting ${ }^{7}$ those variants. Furthermore, any cultural system that has a stronger correlation of this kind than another cultural system will be more adaptive, ceteris paribus. The Fundamental Constraint employs two concepts - the fitness of cultural variants and the fitness impact of those variants - that need explication. The fitness of a cultural variant is based on the probability of the variant being received, implemented, and propagated by the organisms in the population. Some variants will have a higher fitness than others, but this does not mean that those variants are better for the organisms that adopt them. They might simply be easier to implement (perhaps they are less complex), more likely to propagate (perhaps they are more likely to be exhibited in social situations), or more likely to be received (perhaps due purely to the attractiveness of the variant) than an alternate variant. (These are examples of what Boyd and Richerson call biased transmission.) By the fitness impact of cultural variants on organisms, I mean the change in the organism's fitness conferred by the adoption of a particular cultural variant. ${ }^{8}$

There are two kinds of cultural systems: facultative cultural systems and obligate cultural systems. A facultative cultural system is one in which the organisms can abstain from engaging in cultural transmission. If the system is facultative, the zero point on the horizontal axis in Figure 1 represents the organism not adopting a cultural variant (or adopting a variant with no fitness impact). Thus, a point on the left side of the graph represents a cultural variant

6 For a discussion of the importance of these kinds of evaluations in cultural systems, see Castro and Toro (2004).

7 To adopt a variant is to receive and implement it.

8 This of course requires that the fitness of organisms be able to change over time. Although I have argued elsewhere that the fitness property in the theory of natural selection cannot change over time (see Ramsey 2006), I do think that there is a fitness concept (what I call Flux Fitness) that does satisfy this requirement. Flux Fitness is based on an organism's instantaneous probability of surviving and reproducing. If one is troubled by a dynamic fitness concept, the horizontal axis can be reinterpreted to be the difference in fitness between two distinct organisms $O_{1}$ and $O_{2}$ that differ only in their probability of adopting the cultural variant $c$ represented on the vertical access, i.e., the values on the horizontal axis in Figure 1 are $\omega O_{1}-\omega O_{2}$, where $p\left(O_{1}\right.$ accepting $\left.c\right)=1$ and $p\left(O_{2}\right.$ accepting $c$ ) $=0$. 
that is worse for the organism than not adopting any variant, whereas a point on the right represents a variant that is better than adopting no variant at all. An obligate cultural system is one in which the organisms cannot abstain from engaging in cultural transmission; they are forced by their circumstances to adopt one cultural variant or another. An example of such a system is vertical diet inheritance in rats (see Galef 1977). The fetal rats absorb the chemicals from the food consumed by their mother and thereby inherit preferences for this food. This is an obligate system since the rats cannot opt out of this inheritance. Although it is true that rats could evolve to be uninfluenced by the diet of their mother, such a change requires evolutionary change and cannot arise during the development of an individual rat. It is this failure to be able to opt out during development that makes something an example of obligate cultural transmission. For obligate cultural systems, the fitness values represented on the horizontal axis in Figure 1 will be relative to the pool of cultural variants, and the variant that is the worst to adopt can be arbitrarily set to zero. (This means that there will be no negative values on the horizontal axis for obligate systems. This is not a problem, since the important thing is not the absolute value on the horizontal axis, but rather the relationship between the values on the horizontal and vertical axes.)

A variant in the lower left side of the left quadrant (' $a$ ' in Figure 1) is one that is highly deleterious for the individual that adopts it and has a positive (though low) probability of being passed on. A culture of celibacy, drug addiction, or the consumption of a tasty though toxic fruit could occupy point $a$. If such a variant arises, it can be made to conform to the Fundamental Constraint, either by decreasing the $\omega c$ (represented by the solid arrow in Figure 1), increasing the $\Delta \omega O$ (represented by the dashed arrow), or by changing both $\omega c$ and $\Delta \omega O$ in any combination that brings point $a$ closer to the correlation line or decreases $\Delta \omega O$ to 0 . With the fruit example, increasing

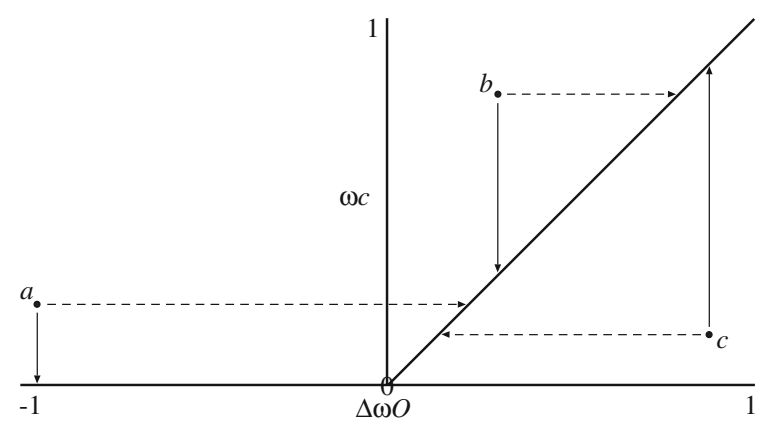

Figure 1. $\omega=$ fitness; $\omega c=$ cultural variant fitness; $\Delta \omega O=$ the fitness impact on the organism of adopting the variant. The diagonal line represents a perfect correlation between $\omega c$ and $\Delta \omega O$. Points $a, b$, and $c$ represent variants that deviate from the correlation line. The arrows illustrate ways of correcting this deviation by a change in $\Delta \omega O$ (the dashed arrows) or $\omega c$ (the solid arrows). The deviation can also be corrected through a change in both $\Delta \omega O$ and $\omega c$ (this is not illustrated). 
the $\Delta \omega O$ could be accomplished by the addition of a method of detoxifying the fruit; decreasing the $\omega c$ could be accomplished by simply refusing to eat the fruit. Variant $b$ is highly likely to be passed on and confers a positive, though moderate, boost to the organism's fitness. Variant $c$ is very good for the organism that adopts it, but has a low fitness and is thus unlikely to spread. An example of variant $c$ is the consumption of a species of insipid but nutritious nut. One way of bringing this variant in line with the correlation line is to increase the desirability of eating this kind of nut, perhaps through a method of processing the nut prior to consumption (this change would be along the solid arrow). If the Fundamental Constraint is satisfied, i.e., if the correlation represented by Figure 1 is maintained, then individuals in the population will tend to adopt the best variants and, for facultative systems, it will tend to be better to adopt a cultural variant then to adopt none at all. If the situation were otherwise, if, say, the slope of the line were negative instead of positive, the worst variants would become the most popular. Additionally, because deleterious variants are bound to arise, these variants, being the fittest, would spread through the population and make it worse to be cultured than not.

In proposing the Fundamental Constraint, I am not claiming that in cultural systems variants must all fall on the correlation line. Rather, the claim is one about the overall structure of the population of cultural variants. It could be the case that for some particular cultural system, the fittest variants are neutral. This is not a problem as long as this deviation from the correlation line is offset by other variants: a cultural system will not be adaptive if all of the variants are neutral. I am claiming, however, that for such a system with the fittest variants being neutral, the system will be more adaptive if the fittest variants are also the most fitness enhancing for the organisms, ceteris paribus.

Now that we have seen that there is a Fundamental Constraint, let's turn to the question of how this constraint can be satisfied.

\section{Ways of satisfying the constraint}

In order to satisfy the constraint, there must be some structure in place, or some force, that engenders and maintains the correlation between the fitness of cultural variants and their fitness impact on the organisms adopting those variants. There are two ways to satisfy the Fundamental Constraint, what I am calling structural solutions and evaluative solutions.

\section{Structural solutions}

Structural solutions are solutions that shape the way in which cultural variants spread through the population, but are not based on any organisms' evaluation of their own fitness, the fitness of others, or the potential fitness benefit of adopting cultural variants. It is commonly believed that a major difference between genetic and cultural systems is that unlike genetic systems, in which organisms do not need to evaluate their own alleles or the alleles of others (in 
order to figure out, say, whether their alleles are better than their neighbor's), ${ }^{9}$ cultural systems require that organisms evaluate their own cultural variants or the variants of conspecifics (in order to determine, for example, which variant is the best one to copy). By pointing out that there are purely structural solutions, I am arguing against this claim. ${ }^{10}$

One structural solution is for all cultural inheritance to occur vertically (i.e., from biological parent to offspring only). There are two kinds of vertical inheritance, one - fixed vertical inheritance - in which the variant is inherited from only one parent and this parent always possesses just one variant. This satisfies the Fundamental Constraint because the only way a cultural variant can become fitter is for it to boost the fitness of the organism that adopts it. An example of this would be offspring learning to forage from their mother. She is presenting them with just one model for how to forage, and the better the foraging strategy, the fitter the individuals will tend to be.

A slightly more complex case - flexible vertical inheritance - is vertical inheritance in which the offspring are exposed to multiple, competing variants from their parents. An example would be both parents acting as models for the foraging strategies of their offspring and each offspring adopting just one of the strategies. In a system such as this, there is the possibility of a worse (for the organism) variant winning out over a better one. (This is analogous to meiotic drive.) If one foraging strategy is simpler, for example, it might be easier to learn than the more complex one. This could confer a higher probability of adoption of the simpler strategy even if the strategy is less profitable. ${ }^{11}$ Although this kind of learning bias can militate against the satisfaction of the Fundamental Constraint, the Fundamental Constraint will still be satisfied if the selection pressure for good foraging strategies swamps the learning bias. And since the individuals with good strategies will tend to be the ones that choose the best of the competing variants, there will be strong selection against these sorts of learning biases. Because of this, vertical transmission systems generally satisfy the constraint. ${ }^{12}$

\footnotetext{
9 In sexual selection, of course, individuals often make mating choices founded on (genetically based) phenotypic trait differences.

10 Although some structural solutions require cognitive sophistication, others clearly do not. Sterelny $(2006,142)$ claims that "... sucking information out of parental minds is not a dumb, blind process." While this is often the case (especially in humans) it need not be. The above example of diet inheritance in rats is an example of this.

11 There is, however, a cost associated with the ability to adopt complex variants. This ability might require larger, more complex nervous systems. Thus, the adoption of a simpler and ostensibly less optimal variant might be more adaptive than having to build bigger brains to make the adoption of more complex variants possible.

12 Genetic inheritance is of course purely vertical (at least for multicellular organisms), and the Fundamental Constraint helps to show why it is good for organisms to possess such a system: if the transmission is vertical and the variants always come from a single parent, or if they come from either parent (but which parent the variant comes from is determined stochastically), then the constraint is automatically satisfied. Although I am considering the Fundamental Constraint only for the case of culture, I would argue that it is a constraint on inheritance mechanisms in general, not just cultural systems.
} 
Horizontal and oblique transmission systems, ${ }^{13}$ on the other hand, do not automatically satisfy the constraint, i.e., a cultural variant can be fit without it conferring a fitness advantage on the organism that adopts it. This does not mean that only systems of vertical transmission satisfy the constraint. There are a number of learning strategies that act as structural solutions for horizontal and oblique transmission systems. There is, in fact, an indefinite number of such strategies, ${ }^{14}$ but many of them are unstable or unlikely to evolve. For a behavioral strategy to count as a structural solution, it cannot require that the organisms make assessments of the fitness of their self or their conspecifics. One such strategy is copy old individuals. This strategy can satisfy the constraint if the variants affect the survivorship of individuals and if once an individual accepts a variant, it replaces this variant only rarely or never. Thus, an individual who copies an old individual will tend to adopt a behavior that has withstood a lifetime of selection pressures and will tend to be better than a variant adopted by an individual who did not make it to old age. It may seem that such a strategy is an evaluative, not a structural solution, but no evaluations need take place in order to realize such a strategy. For example, it could be that young individuals attend school to learn some set of skills and that the teachers are old. The students need not evaluate whether their teachers are old (or the bearers of good variants). The society is simply structured in such a way that the teachers from which the young learn are old.

Another behavioral strategy structural solution is copy individuals of highest rank. This is a solution if the highest ranked individuals tend to be the fittest and the fittest individuals tend to possess the best cultural variants. This also might seem like it is not a structural solution, since it would appear to require discriminations based on rank. But this need not require any assessment of the quality of the self or other. It might just be that the highest ranked individuals occupy a unique spacio-temporal position in the group. For example, if the highest ranked individual occupies the most conspicuous position within the group, a young individual searching for a model will have a high probability of copying this individual relative to other individuals within the group. This difference in probabilities is not based on the discriminative abilities of the copier - it is merely based on the structure of the society. A related solution is referred to as 'prestige-based guided variation' by Henrich and Gil-White (2001). Because there is a correlation between prestige and age (Simmons 1945), the rules copy prestigious individuals and copy old individuals will sanction the copying of many of the same individuals.

Another way to satisfy the constraint is to occasionally opt out of social learning (and instead learn individually) or to socially learn from an individual

13 Horizontal transmission is transmission among individuals in the same generation. Oblique transmission includes all transmission from one generation to the following generation that is not vertical, i.e., that is not from biological parents to offspring.

14 For a more extensive discussion of learning strategies and their adaptiveness, see Laland (2004). 
who opted out of social learning. ${ }^{15}$ The extent to which the Fundamental Constraint is satisfied can depend on the frequency of individual learning. One way of introducing an advantageous variant into the population is to learn something individually and act as a model for another individual. If none of the individuals in a population learn on their own, if all merely copy each other, then the variants will become less and less good for the organisms. This will shift the correlation line in Figure 1 to the left as well as make it tend towards vertical, breaking the connection between the fitness of the variants and their fitness impact on the organisms. This frequency dependence becomes very important in rapidly changing environments. In such environments, cultural variants rapidly lose their benefit to the organisms (see Boyd and Richerson 1985). Thus, we would expect that cultural systems in rapidly changing environments would include a lot of individual learning. Opting out (or copying those who opt out) can be a structural solution - individuals can opt out with some set frequency, not based on their assessments of the quality of the variant or the bearer of the variant - but it can also be an evaluative solution. Individuals can opt out if they judge that the behaviors of the models are suboptimal, for example.

\section{Evaluative solutions}

Evaluative solutions fall into three categories, those that require the evaluation of the self, others, or both. One example of a solution that requires an evaluation of the self is copy if dissatisfied. If an individual correctly assesses that it is not doing well in some domain, it will tend to pay to copy a conspecific's behavior in this domain. A similar strategy is copy if uncertain. If an individual is uncertain how to behave in some domain, copying a conspecific will tend to be profitable. Evaluative solutions can be combined with structural solutions for an even better solution. For example, the compound strategy copy old individuals if uncertain is better than either copy if old or copy if uncertain.

A strategy that requires the evaluation of others (but not the self) is copy successful individuals. This strategy satisfies the constraint only if successful individuals tend to possess success endowing cultural variants. Since this is not guaranteed, the better strategy is copy successful behaviors. It is often a lot harder to determine which behaviors are successful than to determine which individuals are successful. ${ }^{16}$ In the case of humans, it is a lot easier to tell which farmers were successful (simply measure their yield) than to tell what it is that made them successful - was it their seed stock, their fertilization, watering, planting, or harvesting methods, or perhaps some combination of these?

15 This of course can occur only in facultative cultural systems. When an organism opts out of social learning, it is likely to opt out in one or a few domains only. It is not necessary (and often not possible) that it opt out of social learning altogether. Henrich and Boyd (1998) show that occasional use of individual learning is beneficial since it helps in avoiding suboptimal traditions. See Boyd and Richerson (1989) for models relating individual learning, social learning, and the adaptiveness of culture.

16 This is an example of what Boyd and Richerson (1985) refer to as an indirect bias. 
Similarly, it often easier to tell that one group is doing better than another than to identify which individuals in the group are responsible for this success. When someone wins a Nascar race, we can confidently say that the team did well. But what gave the team the winning edge? Was it the driver, the design of the car, the mechanics? This is much more difficult to discern.

A related strategy that requires an evaluation of both others and the self is copy if better. Like the copy if successful strategy, there are two versions, one involves copying the behaviors of individuals that are doing better. The other (more cognitively challenging) version is copy better behaviors.

The above evaluative solutions operate by accepting good variants and expunging bad variants. But there is another way to satisfy the Fundamental Constraint: the adaptive transformation (i.e., guided mutation) of existing variants. The ability to transform variants can be divided into two classes, those based on concrete information and those based on abstract information. I will call these concrete transformations and abstract transformations. Concrete transformations are transformations based on concrete sensory feedback. If a rat eats a food item and subsequently gets sick, it will probably modify its diet to eliminate this kind of food. This is an example of concrete transformation. If, on the other hand, the rat "considers" foraging in a traditional exposed area, "judges" it to be too dangerous, then "decides" to forage only in more protected areas, the rat will have accomplished an abstract transformation. ${ }^{17}$

Another distinction can be drawn between transformations based on proximate feedback and those based on distal feedback. The above example of the rat getting sick following its eating a food item is a case of proximate feedback - the effect quickly follows its cause. If, on the other hand, a rat chooses a mate, there will not be proximate feedback on whether this was a good choice or not - the offspring produced by the pair might be genetically inferior, but even if this inferiority is detected, the connection between this inferiority and the mate is not based on proximate sensations.

Humans are experts at abstract transformations and are able to effect them based not just on distal effects that actually occurred, but on the representations of what might occur. ${ }^{18}$ Although humans can effect such

17 To what extent the rats are able to perform the actions in scare quotes in the previous sentence is questionable. See the arguments in the following section for the rarity of culture.

18 Dennett (1995) makes the distinction between Darwinian creatures - creatures with rigid behavioral responses, Skinnerian creatures - creatures that blindly try different responses, some of which get reinforced, increasing the probability of the reinforced behavior, Popperian creatures creatures with an inner selective environment that can pre-sort the good behaviors from the bad without having to try out the bad behaviors, and Gregorian creatures - the subset of Popperian creatures able to import mind tools to be used in both the generation and testing of behavioral variants. Skinnerian creatures are presumably only able to make concrete transformations, whereas at least some Popperian creatures (and perhaps all Gregorian creatures) are able to make abstract transformations. Just because a creature is able to make abstract transformations does not mean that it can make them in all domains. It is likely that any creature capable of abstract transformations in some domains (such as navigating a hillside) will have domains in which the behavior is rigid or not readily transformable (such as increasing one's pulse in the presence of a predator). 
transformations, part of my argument for the rarity of culture (as we will see below) is that abstract, especially abstract distal, transformations are outside of the cognitive abilities of most species.

\section{The form and distribution of cultural systems}

The solutions presented in the previous section are all ways of filtering out or improving deleterious cultural variants and retaining good ones. This can occur either through the structural or evaluative solutions (or both). Because of this limited domain of solutions to the Fundamental Constraint, one would predict a limited domain of cultural systems in nature. ${ }^{19}$

One way to see why the Fundamental Constraint predicts that culture, especially complex, cumulative culture should be rare is the following argument: cultural transmission is either purely vertical or it is not. If it is purely vertical, then the Fundamental Constraint is automatically satisfied (as we saw above). But this comes at a cost. If the transmission is purely vertical, then (1) cultural inheritance acts like genetic inheritance, which implies that (2) individuals cannot incorporate innovations from any conspecifics except biological parents, (3) good variants cannot spread through the population except through the fecundity of the lineage bearing the variant, and (4) bad variants cannot be replaced by other, better variants from the population, i.e., bad variants can only be eliminated through behavioral plasticity or natural selection on the bearer of the variant. Additionally, (5) if the majority of the cultural mutations are bad (which we would expect unless the variation is directed), then if the cultural system has a slow mutation rate, cultural change is rather slow and it is unlikely that such a cultural system would be favored over a genetic system. If it has a rapid mutation rate, the species would have to have a high fecundity in order to purge the deleterious variants or would have to have a robust system of adaptive transformation. In sum, there is a limited space in which vertical cultural transmission would be favored over genetic transmission.

In spite of these restrictions on vertical cultural transmission, there are cases of adaptive vertical inheritance. Many of these cases involve transformations of some sort (e.g., directed mutation) that increases the adaptiveness of the cultural variants. One example of adaptive vertical transmission is diet inheritance, which occurs in many species from bears to butterflies. One well-studied case is diet inheritance in rats (Galef 1977), which I discussed above. One thing that makes the inheritance of diet in rats adaptive is that the offspring inherit

\footnotetext{
19 Depending upon how 'culture' is defined, one might think that there is either a lot or a little culture in nature. The 'all non genetic transmission' definition of culture makes culture ubiquitous, since cytoplasmic inheritance becomes cultural. But what is undoubtedly rare in nature is complex, cumulative culture. Some have even argued that humans are the only species that exhibits cumulativity (e.g., Tomasello et al. 1993). The Fundamental Constraint and the limited ways that it can be satisfied can help explain why this kind of culture is not more common in nature.
} 
preferences for what the mother is eating during gestation. ${ }^{20}$ This is the diet of an adult and is the result of a mixture of the diet inherited from her mother plus a healthy dose of trial and error learning (concrete transformation - i.e., directed mutation). The trial and error learning presumably eliminated most of the deleterious dietary preferences she inherited as well as updated the diet to the changing environment, thus aligning her fitness with the fitness of her cultural variants (and thereby satisfying the Fundamental Constraint).

If the transmission is not purely vertical, then it incorporates oblique or horizontal transmission (or both). If the system incorporates horizontal transmission, then (1)-(5) are not the case: an individual is potentially able to replace any bad variants it has with better versions from conspecifics, cultural change can be rapid, and the variants received from individuals in the same generation will tend to be more up-to-date. But as with vertical transmission, there is a cost. Along with the benefits of horizontal transmission comes the lack of automatic satisfaction of the Fundamental Constraint. In order for the Fundamental Constraint to be satisfied, structural or evaluative solutions must be in place. Are there structural solutions that satisfy the constraint in the case of horizontal transmission? All of the structural solutions listed above (with the exception of opting out and the possible exception of copy individual of highest rank) make the system effectively into a vertical or oblique system, e.g., a horizontal transmission system that adopts the rule copy old individuals has effectively changed into a vertical/oblique system. Because there are few structural solutions that still allow horizontal transmission, evaluative solutions will generally be needed. A rule like copy if better, if followed, will satisfy the constraint. But what are the cognitive requirements for such a rule? Although this is of course a difficult question to answer, it is probable that for some kinds of variants the cognitive requirements are substantial. The organisms need to resist the seduction of intrinsically attractive variants and adopt new ones only if they are better than what they already have. If proximate feedback is available, concrete transformations can be used to keep the organisms from possessing deleterious variants. But if we include the larger domain of variants with distal or hypothetical effects, a much more subtle and powerful kind of mechanism for sorting good variants from bad is required, something that humans constantly employ, but other species may not have to the degree necessary for them to satisfy the Fundamental Constraint. This sorting mechanism may go a long way toward explaining the rarity of culture in nature and the uniqueness of human complex, cumulative culture.

Oblique transmission represents an intermediate case between vertical and horizontal transmission. If the transmission is oblique only, or if it is oblique and vertical, then the Fundamental Constraint will tend to be satisfied if the variants affect survivorship. An evaluative solution like "copy the best behavior" can work well in an oblique transmission system, since the best variants from each generation can be selected and the worst variants can expire

20 Galef has also shown that there is horizontal transmission of diet in rats (e.g., Galef 1988). 
with the death of their bearers. But oblique transmission also suffers from modified versions of (2)-(5) above, which eliminates a number of the benefits that culture can provide: in oblique systems, individuals cannot incorporate innovations from any conspecifics except individuals from their parent's generation, which means that any good variants that arise must wait until the next generation to spread through the population. If the environment is rapidly changing, what was an adaptive variant a generation ago may no longer be adaptive. Furthermore, without evaluative solutions (like the "copy the best behaviors' example above) variants that are bad for their bearers can become popular (e.g., celibacy). Thus, for many cases, oblique systems will require evaluative solutions in order to satisfy the Fundamental Constraint.

In sum, vertical inheritance systems will generally satisfy the constraint but will rarely be favored over genetic transmission except in systems involving adaptive transformations (directed mutations). Horizontal and oblique systems do not automatically satisfy the constraint and generally require evaluative solutions in order to be adaptive. The rarity of these kind of systems in nature can in part be explained by the rarity of cognitive systems capable of these kinds of evaluations.

\section{Conclusions}

We have seen that there is a Fundamental Constraint on the evolution of culture and that this constraint must be satisfied for a cultural system - or any kind of inheritance system - to be adaptive. This requirement strongly limits the variety of cultural systems that can evolve, and thus helps to explain why there is not more culture in nature. This is the case because the two ways to satisfy the constraint - structural and evaluative solutions - are often either no better than genetic inheritance or require advanced cognitive abilities. Most animal species are expected to be able to perform only concrete transformations. In order to take full advantage of a cultural system, the individuals would need to be able to perform abstract transformations. This points to a key empirical question: What does it take to evolve the ability to make abstract cultural transformations? In the case of the origins of human culture, the central question is: What did it take for our ancestors to evolve the ability to make abstract cultural transformations? Any account of the origins and evolution of culture - human or infrahuman - will have to be an account of how the Fundamental Constraint is satisfied.

\section{Acknowledgements}

I thank Robert Brandon and Alex Rosenberg for reading and commenting on multiple drafts of this paper. I also thank Natalie Gold, David Kaplan Steve Nowicki, Kim Sterelny, Peter Richerson, and an anonymous reviewer for their 
helpful comments and suggestions. A previous version of this paper was presented at the 2005 meeting of the International Society for the History, Philosophy, and Social Studies of Biology.

\section{References}

Boyd R. and Richerson P.J. 1985. Culture and the Evolutionary Process. University of Chicago Press, Chicago.

Boyd R. and Richerson P.J. 1989. Social learning as an adaptation. Lect. Math. Life. Sci. 20: 1-26. Byrne R.W. and Russon A.E. 1998. Learning by imitation: A hierarchical approach. Behav. Brain Sci. 21(5): 667-721.

Castro L. and Toro M.A. 2004. The evolution of culture: From primate social learning to human culture. Proc. Natl. Acad. Sci. USA 101(27): 10235-10240.

Dennett D.C. 1995. Darwin's Dangerous Idea: Evolution and the Meanings of Life. Simon \& Schuster, New York.

Galef B.G. 1988. Communication of information concerning distant diets in a social, central-place foraging species: Rattus Norvegicus. In: Zentall T.R. and Galef B.G. (eds.), Social Learning: Psychological and Biological Perspectives., Lawrence Erlbaum Associates, Hillsdale, pp. 119140.

Galef B.G. 1977. Mechanisms for the social transmission of food preferences from adult to weanling rats. In: Barker L.M., Best M. and Domjan M. (eds.), Learning Mechanisms in Food Selection, Baylor University Press, Waco, TX, pp. 123-150.

Henrich J. 2004. Demography and cultural evolution: How adaptive cultural processes can produce maladaptive losses - the Tasmanian case. Am. Antiq. 69(2): 197-214.

Henrich J. and Boyd R. 1998. The evolution of conformist transmission and the emergence of between-group differences. Evol. Human Behav. 19(4): 215-241.

Henrich J. and Gil-White F.J. 2001. Evolution of prestige: Freely conferred deference as a mechanism for enhancing the benefits of cultural transmission. Evol. Human Behav. 22(3): 165-196.

Laland K.N. 2004. Social learning strategies. Learn. Behav. 32(1): 4-14.

Ramsey G. 2006. Block fitness. Stud. Hist. Philos. Biol. Biomed. Sci. 37: 484-498.

Ramsey G., Bastian M.L. and van Schaik C. Animal innovation defined and operationalized (submitted).

Reader S.M. and Laland K.N. (eds), 2003. Animal Innovation. Oxford University Press, Oxford.

Simmons L.W. 1945. The Role of the Aged in Primitive Society. Yale University Press, London.

Sterelny K. 2006. The evolution and evolvability of culture. Mind Lang. 21(2): 137-165.

Thorpe W.H. 1963. Learning and Instinct in Animals: New ed. Methuen, London.

Tomasello M. 1999. The Cultural Origins of Human Cognition. Harvard University Press, Cambridge, Mass.

Tomasello M., Kruger A.C. and Ratner H.H. 1993. Cultural learning. Behav. Brain Sci. 16(3): 495552.

Whiten A. and Ham R. 1992. On the nature and evolution of imitation in the animal kingdom. Adv. Study Anim. Behav. 21: 239-283. 